EPJ Web of Conferences 59, 14006 (2013)

DOI: $10.1051 /$ epjconf/20135914006

(C) Owned by the authors, published by EDP Sciences, 2013

\title{
Effect of autoionizing states with a high-lying spectator electron on population inversion of nitrogen plasma in recombining phase
}

\author{
T. Ozawa, H. Kumai, E. Hotta, K. Horioka and T. Kawamura \\ Tokyo Institute of Technology, Department of Energy Sciences, Nagatsuta 4259, Midori, \\ Yokohama, Kanagawa, Japan
}

\begin{abstract}
Discharge produced plasma (DPP) is a promising tool to generate effective extreme ultraviolet (EUV) light. A hydrogen-like nitrogen ion emits light at $13.4 \mathrm{~nm}$ with an optical allowed atomic transition from $n=3$ to 2 , where $n$ stands for a principal quantum number. We focus on the properties of population inversion between $n=3$ and 2 in recombining phase. Population inversion is calculated in the framework of population kinetics, and a gain coefficient $G$ is estimated. In this study, dielectronic-capture ladderlike (DL) processes are included. At high density and low temperature, the DL deexcitations make a large contribution to the population inversion.
\end{abstract}

\section{INTRODUCTION}

A short-wavelength light source has been used for such industrial applications as nanostructure science and technology [1]. Extreme ultraviolet (EUV) light is one of the most promising candidates for the next generation lithography. The wavelength of EUV light is typically $5 \sim 40 \mathrm{~nm}$, and can be obtained by creation of hot dense plasma. The EUV light generation has been experimentally done by intense laser light and/or high current discharge [2,3]. In general, hot dense plasma creation by discharge seems to be more efficient due to its compact and simple scheme compared with that by laser irradiation [4].

In this study, hydrogen-like nitrogen is chosen as one of EUV light emitters of which wavelength is $13.4 \mathrm{~nm}$. The wavelength is near that for the next generation semiconductor lithography, and may be expected to have potential for the industrial use described above. The $13.4 \mathrm{~nm}$ light comes from an optical allowed transition from $n=3$ to 2 (Balmer- $\alpha$ ). To get enough intensity of the radiation, nitrogen must be ionized up to the ionization degree of six. In a recombining scheme, hot dense fully stripped nitrogen plasma is created and the hydrogen-like nitrogen is populated by three body recombination with rapid plasma expansion and cooling, resulting in the creation of population inversion and the lasing of Balmer- $\alpha$. Although a related experiment has been performed and a pulse waveform shaping is designed for the rapid cooling, there is no lasing [5]. A related magnetohydrodynamic (MHD) simulation using an atomic population kinetics code for Z-pinch recombination pumping was performed [6]. In the research, a recommended pressure is estimated and the maximum gain of $0.11 \mathrm{~cm}^{-1}$ is obtained. However, because of plasma motion, understanding of the lasing characteristics in the viewpoint of atomic population kinetics is not clear. We calculate population kinetics with stepwise cooling to gain an insight into the lasing of nitrogen at $13.4 \mathrm{~nm}$.

In the calculation, doubly excited states whose outermost electron is bound in a higher orbital are considered. Those states can be implicitly included in an atomic model proposed by Fujimoto et al . [7]. The processes in the model are called dielectronic-capture ladderlike (DL) processes. Such atomic

This is an Open Access article distributed under the terms of the Creative Commons Attribution License 2.0, which permits unrestricted use, distribution, and reproduction in any medium, provided the original work is properly cited. 


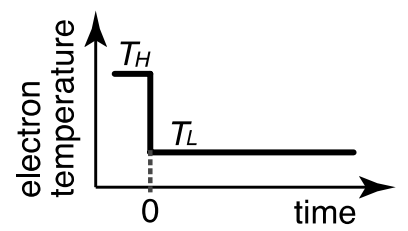

Figure 1. Time evolution of electron temperature.

processes are not considered in the study done by Vrba et al. [6]. In a recombining plasma scheme, the DL deexcitation may play an important role to create the population inversion.

\section{ESTIMATION OF LASING PERFORMANCE}

The usual goal for lasing has been to achieve a gain-length product $G L=5$. With the cross-section of stimulated emission $\sigma_{\text {stim, }}$, the gain coefficient $G$ of Balmer- $\alpha$ is

$$
G=N_{\mathrm{n}=3} \sigma_{\text {stim }}\left[1-\left(N_{\mathrm{n}=2} / g_{\mathrm{n}=2}\right) /\left(N_{\mathrm{n}=3} / g_{\mathrm{n}=3}\right)\right],
$$

$N_{\mathrm{n}=2}$ and $N_{\mathrm{n}=3}$ are the population density of $n=2$ and 3 , respectively. $g_{\mathrm{n}=2}$ and $g_{\mathrm{n}=3}$ are the corresponding statistical weights. To estimate $\sigma_{\text {stim }}$, a spectral shape function is needed. To obtain the shape function, electron impact broadening using a semiclassical expression [8], Stark broadening [9], natural and Doppler broadenings are convolved by a Voigt profile. Because of a time-dependent property of population inversion, a time-averaged gain $G_{\text {ave }}$ is estimated, namely, $G_{\text {ave }}=\int_{0}^{\tau} G d t / \tau$, where $\tau$ is the time at $G=G_{\max } / e$ after reaching $G=G_{\max }$.

\section{MODEL OF POPULATION KINETICS}

In the study by Vrba et al. [6], the kinetic code FLY [10] was used. However, doubly excited states whose outermost electron is bound in a higher orbital are not considered. Fujimoto et al. [7] gives the way to deal with the excitation and deexcitation that have doubly excited or autoionizing states as intermediate states. These processes are called dielectronic-ladderlike (DL) processes. The DL processes are interpreted as an extrapolation of a collisional excitation cross section of a hydrogen-like ion [11] below the excitation threshold down to the Griem's limit [7]. FLYCHK [12] has almost same helium-like doubly excited states as seen in FLY for the elements with $\mathrm{Z}<27$.

\subsection{Simulation conditions}

Focused is a recombining phase after the maximum compression relevant to discharge produced plasma (DPP). To demonstrate the recombining phase, atomic processes are calculated with rapid plasma cooling shown in Figure 1. This cooling condition is expected to give us strong non-equilibrium properties on atomic processes. The ion density is kept constant in the calculation. Since the plasma cooling is assumed to be stepwise to exclude the effect of plasma motion, we can see the property of the population inversion in the viewpoint of population kinetics.

\section{SIMULATION RESULTS AND DISCUSSION}

Figure 2 shows the population densities of hydrogen-like nitrogen ions per statistical weights $\left(N_{\mathrm{n}} / g_{\mathrm{n}}\right)$ without the DL processes. The initial electron temperature $T_{\mathrm{H}}$ is assumed to be $300 \mathrm{eV}$, and the final one $T_{\mathrm{L}}$ is $10 \mathrm{eV}$. The ion densities $n_{\mathrm{i}}$ are $10^{17}$ and $10^{18} \mathrm{~cm}^{-3}$, respectively. Population inversion is created, namely, $N_{\mathrm{n}=3} / g_{\mathrm{n}=3}>N_{\mathrm{n}=2} / g_{\mathrm{n}=2}$. The time-averaged gain $G_{\text {ave }}$ is mostly lower than $10^{-2} \mathrm{~cm}^{-1}$ 

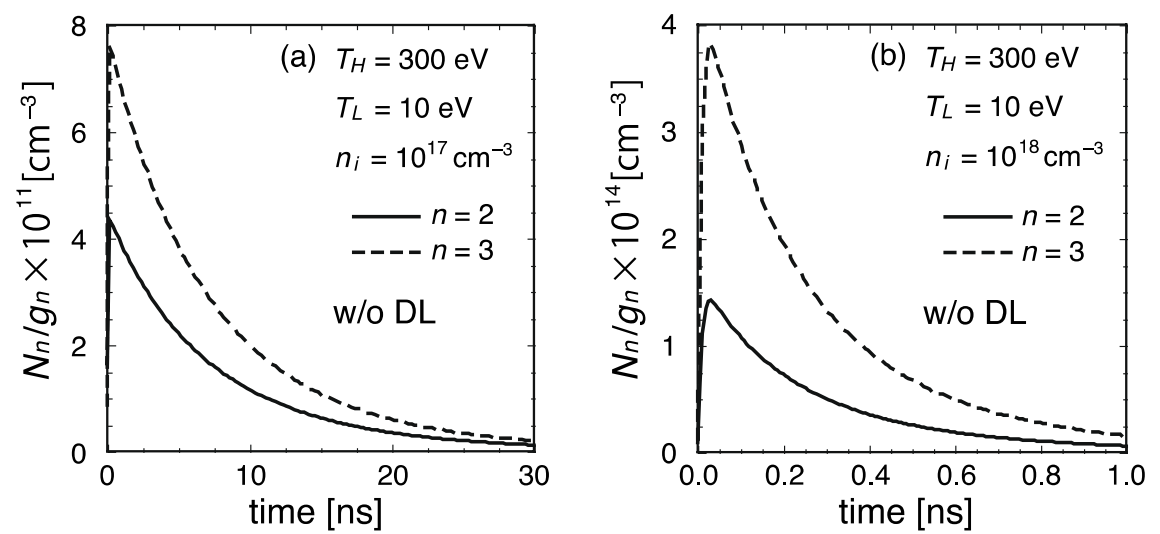

Figure 2. Population densities of hydrogen-like nitrogen ions per statistical weights $(n$ stands for a principal quantum number). (a) $n_{\mathrm{i}}=10^{17} \mathrm{~cm}^{-3}$ and (b) $n_{\mathrm{i}}=10^{18} \mathrm{~cm}^{-3}$.
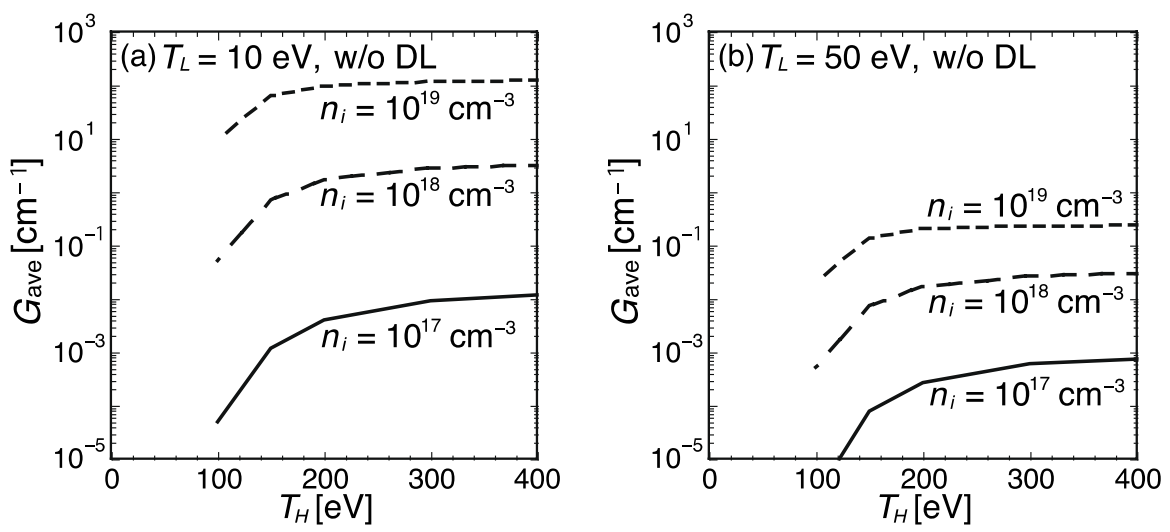

Figure 3. Time-averaged gain at (a) $T_{\mathrm{L}}=10 \mathrm{eV}$ and (b) $T_{\mathrm{L}}=50 \mathrm{eV}$.

at $n_{\mathrm{i}}=10^{17} \mathrm{~cm}^{-3}$, and $G_{\text {ave }}$ is $\sim 2.8 \mathrm{~cm}^{-1}$ at $n_{\mathrm{i}}=10^{18} \mathrm{~cm}^{-3}$ (see Figure 3). The higher $n_{\mathrm{i}}$ is, the larger is $G_{\text {ave }}$. The duration of the population inversion $\tau$ becomes short since a collision between an ion and an electron becomes frequent with increase in the ion density. The plasma length $L$ can be approximately estimated by the product of $\tau$ and the speed of light $c$, and $L$ is $\sim 8.4 \mathrm{~cm}$ at $n_{\mathrm{i}}=10^{18} \mathrm{~cm}^{-3}$. Since $L$ is $\sim 0.24 \mathrm{~cm}$ at $n_{\mathrm{i}}=10^{19} \mathrm{~cm}^{-3}, L$ may be enough when $n_{\mathrm{i}} \leq 10^{18} \mathrm{~cm}^{-3}$.

The dependence of the population inversion on the ion density and electron temperature is shown in Figure 3. These calculations do not also include the DL processes. The lower $T_{\mathrm{L}}$ is, the larger is $G_{\text {ave }}$. With $T_{\mathrm{L}} \geq 100 \mathrm{eV}, T_{\mathrm{H}}$ must be greater than $300 \sim 400 \mathrm{eV}$ to create population inversion. However, as shown in Figure $3, T_{\mathrm{H}}$ can be $\sim 200 \mathrm{eV}$ with $T_{\mathrm{L}}<100 \mathrm{eV}$.

Comparison between the cases with and without the DL processes shows the clear difference with $n_{\mathrm{i}}=10^{17 \sim 18} \mathrm{~cm}^{-3}$ and $T_{\mathrm{L}}=10 \mathrm{eV}$. The results are shown in Figure 4. Since the DL deexcitation from $n=4$ to 3 is remarkable at $n_{\mathrm{i}}=10^{17} \mathrm{~cm}^{-3}$, this process make a contribution to the population inversion between $n=2$ and 3. On the other hand, at $n_{\mathrm{i}}=10^{18} \mathrm{~cm}^{-3}$, the DL deexcitations from $n=4$ to 2 and from $n=3$ to 2 make a large population in $n=2$ and reduce $G_{\text {ave }}$ by about $42 \%$. The calculation of DL processes is broken with $n_{\mathrm{i}} \geq 10^{19} \mathrm{~cm}^{-3}$ and $T_{\mathrm{L}}=10 \mathrm{eV}$ since the large extrapolation of the cross-section is impossible. However, the reduction of the gain due to the DL deexcitations with 

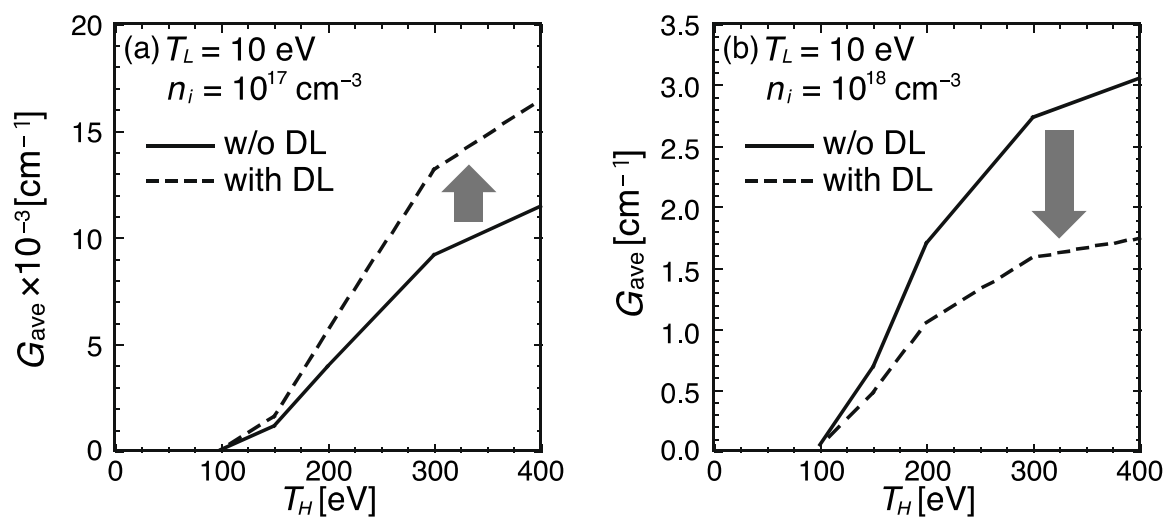

Figure 4. Time-averaged gain with and without DL processes at (a) $n_{\mathrm{i}}=10^{17} \mathrm{~cm}^{-3}$ and (b) $n_{\mathrm{i}}=10^{18} \mathrm{~cm}^{-3}$.

$n_{\mathrm{i}} \geq 10^{19} \mathrm{~cm}^{-3}$ may be larger than that at $n_{\mathrm{i}}=10^{18} \mathrm{~cm}^{-3}$. Then, $G_{\text {ave }} L$ is found to be larger than 5 when $T_{\mathrm{L}}=10 \mathrm{eV}$ and $T_{\mathrm{H}} \geq 200 \mathrm{eV}$, or $T_{\mathrm{L}}=50 \mathrm{eV}$ and $T_{\mathrm{H}}>\sim 250 \mathrm{eV}$ at $n_{\mathrm{i}}=10^{18} \mathrm{~cm}^{-3}$.

\section{CONCLUSIONS}

To get an optimum condition for the generation of $13.4 \mathrm{~nm}$ laser light relevant to discharge produced nitrogen plasma, the population inversion and the gain coefficient of Balmer- $\alpha$ in recombining phase are examined. In this study, we estimate the contribution of DL processes to the population inversion. With $n_{\mathrm{i}} \geq 10^{18} \mathrm{~cm}^{-3}$ and $T_{\mathrm{L}} \sim 10 \mathrm{eV}$, the DL deexcitation make a large contribution, resulting in the reduction of the gain. The processes are important for accurate estimation of the gain. Calculations show that $n_{\mathrm{i}} \sim 10^{18} \mathrm{~cm}^{-3}$ is a good candidate for the lasing of EUV light. Despite the fact that stepwise cooling leads to non-equilibrium atomic physics, the resultant $G_{\text {ave }} L$ may be severe for a practical use. The prescription of plasma cooling is definitely critical.

\section{References}

[1] D. T. Attwood, Soft X-rays and extreme ultraviolet radiation (Cambridge University Press, 1999)

[2] D. L. Matthews, et al., Phys. Rev. Lett. 54, 110 (1985)

[3] M. C. Marconi, J. J. Rocca, Appl. Phys. Lett. 54, 2180 (1989)

[4] J. J. Rocca, et al., Phys. Rev. Lett. 73, 2192 (1994)

[5] Y. Sakai, et al., J. Appl. Phys. 107, 083303 (2010)

[6] P. Vrba, N. A. Bobrova, P. V. Sasorov, M. Vrbova, J. Hubner, Phys. Plasmas 16, 073105 (2009)

[7] T. Fujimoto, T. Kato, Phys. Rev. A 32, 1663 (1985)

[8] H. Nguyen, M. Koenig, D. Benredjem, M. Caby, and G. Coulaud, Phys. Rev. A 33, 1279 (1986)

[9] G. D. Pollak, N. D. Delamater, J. K. Nash, B. A. Hammel, J. Quant. Spectrosc. Radiat. Transf. 51, 303 (1994)

[10] R. W. Lee, J. T. Larsen, J. Quant. Spectrosc. Radiat. Transf. 56, 535 (1996)

[11] V. I. Fisher, Y. V. Ralchenko, V. A. Bernshtam, A. Goldgirsh, Y. Maron, Phys. Rev. A 55, 329 (1997)

[12] http://nlte.nist.gov/FLY/Doc/Manual_FLYCHK_Nov08.pdf, p.44 and p.57 (2008) 